\title{
OPTIMIZING EXISTING TOOLS FOR REACHING AN ADEQUATE DISEASE CONTROL IN PATIENTS WITH SPONDYLOARTHRITIS
}

\author{
Claudia Deaconu ${ }^{1}$, Daniela Opris ${ }^{1,2}$, Diana Mazilu ${ }^{1,2}$, Laura Groseanu ${ }^{1,2}$, Andreea Borangiu ${ }^{1,2}$, \\ Saulescu Ioana ${ }^{1,2}$, Alexandra Peltea ${ }^{1}$, Magdalena Negru ${ }^{1,2}$, Cosmin Constantinescu ${ }^{1,2}$, Violeta Vlad ${ }^{1}$, \\ Violeta Bojinca $^{1,2}$, Andra Balanescu ${ }^{1,2}$, Denisa Predeteanu ${ }^{1,2}$, Ruxandra Ionescu ${ }^{1,2}$ \\ ${ }^{1}$ Department of Rheumatology and Internal Medicine, Sfanta Maria Clinical Hospital Bucharest, Romania \\ ${ }^{2}$ Carol Davila University of Medicine and Pharmacy, Bucharest, Romania
}

\begin{abstract}
Background/Objective. Disease activity in spondyloarthritis $(\mathrm{SpA})$ is evaluated through conventional scores such as BASDAI or ASDAS. The aim of this study was to determine disease activity in SpA patients under biological therapy with further assessment on the quality of each activity indicator and their clinical value together with their impact on NSAID intake.

Materials and methods. Over nine months (January-August 2015) we enrolled 100 patients with SpA on antiTNF therapy (33\% IFX; 35\% ADL; 32\% ETA). We collected demographic, clinical (BASDAI, ASDAS, PtGA) and laboratory data (ESR, CRP). Frequency of NSAID ingestion expressed as days per week was included in a questionnaire. Considering their biologic treatment, patients were divided into disease activity groups and sample characteristics were correlated and compared. Statistical analysis was performed with SPSS 20.0, using specific tests such as Pearson, Spearman or ANOVA.

Results. Patients with active disease, stated by both activity scores had an increased NSAID intake. Out of $86 \%$ of patients with a BASDAI under 4 , only $82.6 \%$ had an ASDAS-CRP lower than 1.3. BASDAI correlated to both ASDAS scores $(r=0.65$ and $0.71, P<0.001)$ and PtGA correlated to both disease activity scales, with a stronger correlation to BASDAI $(r=0.912, P<0.01)$ than ASDAS ( $r=0.67$ and $0.71, P<0.01$ for both).

Conclusion. We proved that disease activity scores have good discriminatory power, with a strong correlation between the subjective BASDAI and PtGA. However, ASDAS-CRP proves to be more rigorous than other variables when targeting high and low disease activity, thus indicating the need of an objective component in assessing these patients.
\end{abstract}

Keywords: spondyloarthritis, disease activity scores, discriminatory power, anti-TNF therapy

Abbreviations: IFX-infliximab, ADL-adalimumab, ETA-etanercept, BASDAI-Bath Ankylosing Spondylitis Disease Activity Index, ASDAS-Ankylosing Spondylitis Disease Activity Score, TNF-tumor necrosis factor, SpA-spondyloarthritis, NSAID-non-steroidal anti-inflammatory drugs, ELISA-enzyme linked immunosorbent assay, ESR-erythrocyte sedimentation rate, CRP-C reactive protein, PtGA-patient global assessment, MRI-magnetic resonance imaging, CI-confidence interval, SD-standard deviation, SMD-standardized mean difference, AUC-area under curve, ROC-receiver operating characteristic.

\section{INTRODUCTION}

Spondyloarthritides $(\mathrm{SpA})$ reside in the heterogeneous group of chronic, inflammatory diseases that primarily affect the spine and peripheral joint structures (1). Since the issuing of the new ASAS (Assessment of Spondyloarthritis International Society) classification criteria in 2009, we group patients according to the predominant disease target - either axial, ranging from non-radiographic SpA to ankylosing spondylitis (AS) or peripheral form (2-4).
Since their arrival, anti-TNF (tumor necrosis factor) agents such as adalimumab (ADL), infliximab (IFX) or etanercept (ETA) have become the mainstay treatment in SpA by improving patients' signs and symptoms and lowering overall disease activity $(1,5)$.

Disease activity in $\mathrm{SpA}$ is widely evaluated through disease activity scores such as BASDAI (Bath Ankylosing Disease Severity Index) or ASDAS (Ankylosing Spondylitis Disease Activity Score),

Correspondence address:

Claudia Deaconu, Department of Rheumatology and Internal Medicine, Sf. Maria Clinical Hospital 37-39 Ion Mihalache Bd, District 1,

Bucharest

E-mail: claudiadeaconu1@yahoo.com 
with no proven superiority between the two 6,7. The ASDAS score consists of three questions elected from BASDAI, a PtGA (patient global assessment) and the inflammatory markers (either ESR or CRP).

The aim of this study was to evaluate the clinical quality and discriminating power between the above indices when subdividing patients with anti-TNF therapy according to their disease status indicated by BASDAI, ASDAS or PtGA.

\section{MATERIALS AND METHODS}

\section{Patient recruitment and data collection}

The present study type was prospective, observational and cross-sectional. Over a period of nine months (January - August 2015), 117 patients with established SpA according to the New York modified criteria or the ASAS classification criteria were enrolled in the study. Patients with both axial and peripheral forms were included, with no discrimination in further statistical analysis. No patient in the study group had supplementary synthetic DMARD (disease modifying antirheumatic drug).

The inclusion criteria were based on continuous anti-TNF therapy with one of the agents - ADL, IFX or ETA in the past six months. Criteria of exclusion consisted of discontinuation or delay in treatment administration in the last three months or intraarticular glucocorticoid administration more recent than six weeks.

Demographic data was collected by the same physician together with clinical (disease activity scores BASDAI, ASDAS and PtGA) and laboratory parameters (inflammatory markers ESR erythrocyte sedimentation rate and CRP $C$-reactive protein). The laboratory reference for ESR values ranges from 2 to $20 \mathrm{~mm} / \mathrm{h}$ and for CRP between 0 to $5 \mathrm{mg} / \mathrm{L}$.

As cited by literature guidelines $(8,9)$, we considered a BASDAI score of 4 or greater to indicate an active disease status with a suboptimal control of the disease. According to the ASAS group recommendations (10), an ASDAS score of less than 1.3 corresponds to an inactive disease, greater or equal to 1.3 and less than 2.1 defines a moderate disease activity, ASDAS values between 2.1 and 3.5 relates to high disease activity, whereas ASDAS greater than 3.5 reflects a very high disease activity.

We evaluated patients' global assessment using the PtGA score and setting a cut-off level of over 5 for high disease activity and values lower than 5 for low disease activity (11). This score represents pa- tient's grading of his disease using a visual analogue scale (VAS, 0 not active ranging to 10 extremely active).

\section{Statistical analysis}

The statistical analysis was performed using the SPSS statistical software, version 20.0, setting a standardized significant $\mathrm{P}$ value at 0.05 . Data was expressed as mean value \pm standard deviation (SD). Differences between groups were recorded with the aid of Student t-test, whereas Spearman and Pearson tests were used for correlations. ANOVA test was used to analyze the differences among group means and variations between them.

In order to compare the discriminating ability of the composite indices regarding disease activity we used the standardized mean difference (SMD) with values showing good $(>0.80)$, moderate $(0.50-0.80)$ or small $(<0.50)$ discriminating power. Thus, the higher the value the greater the discriminating capacity (12).

We calculated the area under the curve (AUC) for indices of disease activity. Values superior to 0.90 stand for high accuracy, from 0.70 to 0.90 for moderate accuracy, and from 0.50 to 0.70 for poor accuracy (13).

\section{RESULTS}

We enrolled $117 \mathrm{SpA}$ patients and after applying the inclusion/exclusion criteria mentioned in the prior method section, 17 patients were excluded from the study group due to criteria non-fulfillment. Thirty-three percent of patients were treated with IFX, $35 \%$ of them with ADL and the remaining $32 \%$ had ETA as anti-TNF therapy. Seventy-eight percent were of male gender and 93\% were HLA B27 positive. The mean disease duration was 97.29 months \pm 74.7 SD (standard deviation), as represented in Fig. 1. All patients included in the present study were Caucasian by race.

For the ADL cohort we performed patient distribution according to disease activity status, taking into account both BASDAI and ASDAS scores. Out of the $35 \mathrm{SpA}$ patients with ADL therapy, $12 \%$ were classified as having a high disease activity with a BASDAI greater than 4. Mean BASDAI for the "inactive group" was $1.21 \pm 1.19$, while the highly active had a mean score of $6.57 \pm 2.52$. We noted significant differences in all disease activity indicators (ASDAS, acute phase reactants) Table 1. 


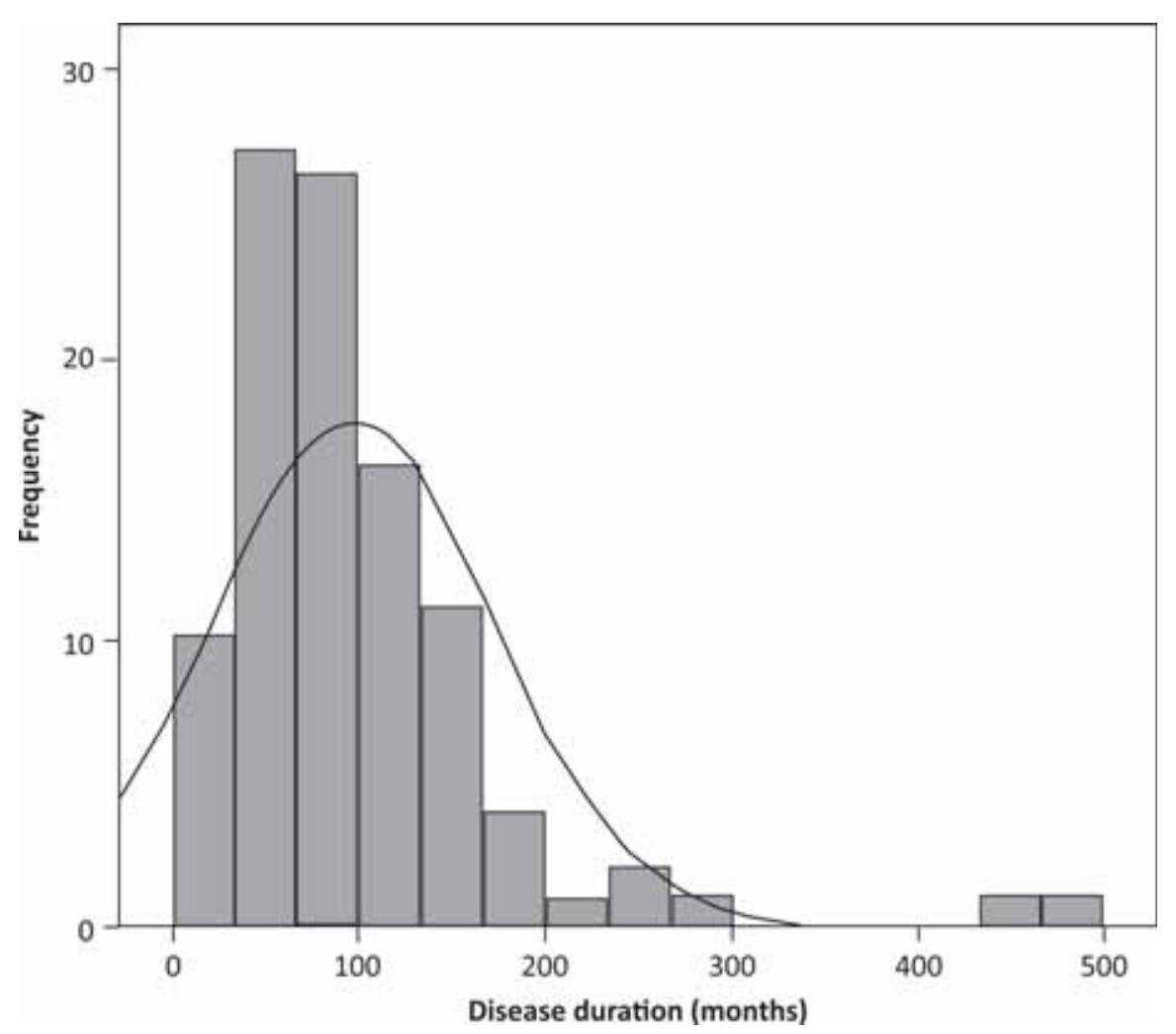

FIGURE 1. Disease duration (months) in study lot
Mean disease duration was slightly higher in patients with active disease, although the difference did not reach statistical significance (119.5 months versus 102.2 in patients with BASDAI lower than 4, $\mathrm{P}=0.71)$.

TABLE 1. Comparison in disease activity indices between ADL patients with high and low disease activity

\begin{tabular}{|c|c|c|c|}
\hline Variable & $\begin{array}{c}\text { High disease } \\
\text { activity }(B A S D A />4)\end{array}$ & $\begin{array}{c}\text { Low disease } \\
\text { activity }(B A S D A /<4)\end{array}$ & $\mathbf{P}$ \\
\hline ASDAS-CRP & $4.08 \pm 1.77$ & $1.29 \pm 0.81$ & $<0.01$ \\
\hline ASDAS-ESR & $3.85 \pm 1.53$ & $1.19 \pm 0.65$ & $<0.01$ \\
\hline CRP $(\mathrm{mg} / \mathrm{l})$ & $54.76 \pm 36.01$ & $7.54 \pm 10.1$ & $<0.01$ \\
\hline ESR $(\mathrm{mm} / \mathrm{h})$ & $50 \pm 33.17$ & $9.48 \pm 8.52$ & $<0.01$ \\
\hline
\end{tabular}

Interestingly, patients with active disease measured through BASDAI score had increased daily NSAID ingestion $(\mathrm{P}=0.02)$.

The structure of ASDAS-differenciated categories among SpA patients undergoing ADL treatment is as follows: 23 patients were considered as having an inactive disease status based on their ASDASCRP calculated score (mean 0.87 \pm 0.29 ), while 5 patients had a moderately active disease. Three patients belonged to the highly active disease and 4 out of the total of 35 patients were labeled with very high disease activity, as seen below in Table 2.

NSAID consumption was significantly higher in patients with higher ASDAS-CRP values $(\mathrm{P}<0.01)$,
TABLE 2. Mean disease activity scores in ADL-treated spondyloarthritis patients (disease activity categories $0=$ inactive; 1 =moderate; $2=$ high disease activity; 3=very high disease activity)

\begin{tabular}{|r|c|r|r|r|}
\hline \multirow{2}{*}{} & \multicolumn{2}{|c|}{ ASDAS-CRP } & \multicolumn{2}{c|}{ BASDAI } \\
\cline { 2 - 5 } & Mean & Std. Deviation & Mean & Std. Deviation \\
\hline 0 &, 875238 &, 2926537 &, 738095 &, 8015461 \\
\hline 1 & 1,760000 &, 2115420 & 2,020000 & 1,353139 \\
\hline 2 & 2,716667 &, 2818392 & 3,536667 &, 4647939 \\
\hline 3 & 4,622500 &, 7891081 & 6,350000 & 2,8219379 \\
\hline
\end{tabular}

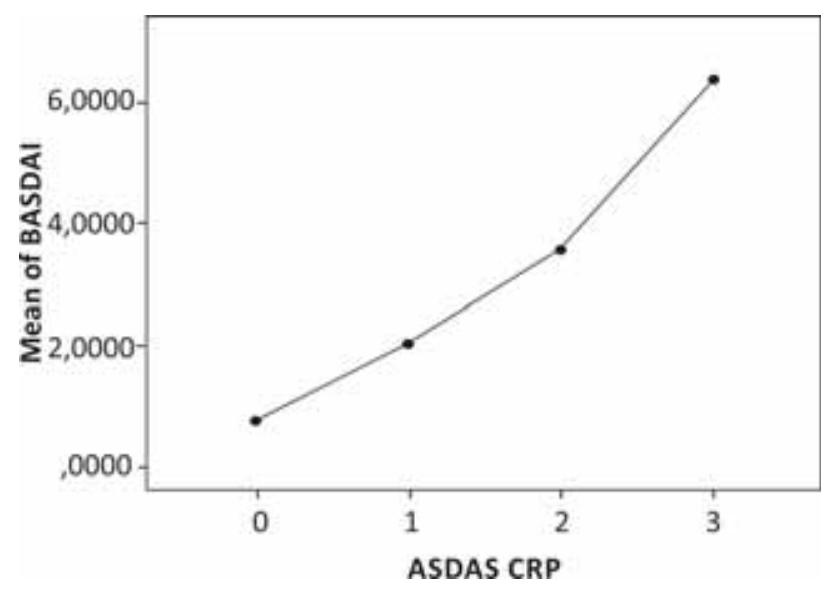

with a mean frequency set at two days per week for patients in the last group (very high disease activity).

Using the BASDAI cut-off of 4, we divided the ETA treated patients into low and high activity subgroups. However, results could not be interpreted because there was only one patient with BASDAI over 4 . 
Further we divided patients on ETA upon ASDAS activity categories according to the ASAS group approved distribution (see Table 3). Thirteen patients corresponded to the inactive group (score $<1.3$ ) with a mean ASDAS-CRP of $0.87 \pm 0.3,12$ had a moderate disease activity (mean of $1.63 \pm 0.2$ ). The high disease activity group comprised of 6 patients with a mean score of $2.63 \pm 0.2$ and only one patient was categorized as having a very high disease activity.

Differences between the BASDAI and ASDAS scores detailed below were not significant $(\mathrm{P}=0.56)$.

TABLE 3. Comparison between mean disease activity scores in ETA-treated patients

\begin{tabular}{|c|c|c|c|}
\hline $\begin{array}{c}\text { ASDAS disease } \\
\text { activity category }\end{array}$ & $\mathbf{N}$ & $\begin{array}{c}\text { ASDAS-CRP } \\
\text { (mean } \pm \text { SD) }\end{array}$ & $\begin{array}{c}\text { BASDAI } \\
\text { (mean } \pm \text { SD) }\end{array}$ \\
\hline Inactive & 13 & $0.87 \pm 0.3$ & $0.76 \pm 0.6$ \\
\hline Moderate & 12 & $1.63 \pm 0.2$ & $1.5 \pm 0.7$ \\
\hline High & 6 & $2.63 \pm 0.2$ & $2.05 \pm 0.42$ \\
\hline Very high & 1 & 3.7 & 4.2 \\
\hline
\end{tabular}

The IFX treated group gathered a total of 33 eligible patients. Patients were subgrouped according to BASDAI cut-offs in high and low disease activity categories (see Table 4). Twenty-one percent were found as having an insufficiently controlled disease, with a mean BASDAI score of 5.07 \pm 1.18 .

TABLE 4. Differences in disease activity between patients with detectable and undetectable IFX serum levels

\begin{tabular}{|l|c|c|c|}
\hline Variable & $\begin{array}{c}\text { High disease } \\
\text { activity }(B A S D A />4)\end{array}$ & $\begin{array}{c}\text { Low disease } \\
\text { activity }(B A S D A /<4)\end{array}$ & $\mathbf{P}$ \\
\hline ASDAS-CRP & $2.98 \pm 1.12$ & $1.47 \pm 0.74$ & $<0.01$ \\
\hline ASDAS-ESR & $3.08 \pm 1.07$ & $1.62 \pm 0.87$ & $<0.01$ \\
\hline CRP $(\mathrm{mg} / \mathrm{l})$ & $40.36 \pm 53.83$ & $4.79 \pm 5.05$ & $<0.02$ \\
\hline ESR $(\mathrm{mm} / \mathrm{h})$ & $40.86 \pm 32.13$ & $14.81 \pm 16.36$ & $<0.05$ \\
\hline
\end{tabular}

NSAID consumption was increased in IFX patients with active disease: approximately 4 days per week in contrast to the other group 1.5 days per week $(\mathrm{P}=0.1)$.

The division of ASDAS-differenciated categories among SpA patients on IFX therapy is the following: 13 patients were considered as having an inactive disease status based on their ASDAS-CRP calculated score, while 10 patients had a moderately active disease. Six patients were classified as suffering from highly active disease and 4 out of the total of 33 patients were categorized as very high disease activity.

Using a crosstabulation task, we noted that out of $86 \%$ of patients with a BASDAI under 4 suggesting a well controlled disease, only $82.6 \%$ had an ASDAS-CRP lower than 1.3, thus indicating that the latter is a more restrictive and rigorous score in assessing disease activity status.

We evaluated disease activity for the entire study group of 100 patients based on conventional scores: BASDAI, ASDAS-CRP, ASDAS-ESR, acute phase reactants and PtGA.

When used as an external criterion PtGA (patient global assessment) showed that $12 \%$ of patients had active disease with a PtGA of over 5 while $88 \%$ were classified as low disease activity $(\mathrm{PtGA}<5)$. Mean ASDAS-CRP and ASDAS-ESR in the active group were 3.39 and 3.24 , respectively as seen below in Table 5. Mean BASDAI score in the high activity group according to PtGA was 5.66.

We showed that both ASDAS scores had good discriminating capacities, with similar values when using the SMD [ASDAS-CRP - SMD 2.0034 (95\% CI 1.2909-2.716) and ASDAS-ESR - SMD 2.0034 (95\% CI 1.2909-2.716)]. In our study group, based on PtGA, BASDAI outperformed ASDAS scores with a SMD of 3.3391 (95\% CI 2.5334-4.1447).

TABLE 5. Disease activity scores in patients with high and low disease activity according to PtGA

\begin{tabular}{|l|c|c|c|}
\hline Variable & $\begin{array}{c}\text { High disease } \\
\text { activity } \\
(\mathrm{PtGA}>5, \mathrm{n}=12)\end{array}$ & $\begin{array}{c}\text { Low disease } \\
\text { activity } \\
(\mathrm{PtGA}<5, \mathrm{n}=88)\end{array}$ & $\mathbf{P}$ \\
\hline BASDAI & $5.66 \pm 1.91$ & $1.34 \pm 1.31$ & $<0.001$ \\
\hline ASDAS-CRP & $3.39 \pm 1.31$ & $1.44 \pm 0.8$ & $<0.001$ \\
\hline ASDAS-ESR & $3.24 \pm 1.35$ & $1.44 \pm 0.75$ & $<0.001$ \\
\hline CRP $(\mathrm{mg} / \mathrm{l})$ & $34.91 \pm 29.11$ & $8.75 \pm 18.86$ & $<0.001$ \\
\hline ESR $(\mathrm{mm} / \mathrm{h})$ & $40.6 \pm 29.29$ & $14.56 \pm 16.5$ & $<0.001$ \\
\hline
\end{tabular}

We used ROC curves of the disease activity scores by using the PtGA $\geq 5$ as variable of high disease activity state. For ASDAS-CRP, ASDAS-ESR and BASDAI the AUCs (area under curve) were 0.89 ( $\mathrm{P}=0.05 ; 95 \%$ CI 0.80-0.99), 0.88 ( $\mathrm{P}<0.001$; 95\% CI 0.77-0.99), and $0.99(\mathrm{P}=0.009 ; 95 \% \mathrm{CI}$ 0.97-1.00), respectively. For CRP and ESR the AUCs were $0.81(\mathrm{P}=0.001 ; 95 \%$ CI $0.64-0.97)$ and 0.79 ( $\mathrm{P}=0.003 ; 95 \%$ CI $0.61-0.96)$, respectively. This shows the high accuracy of the three scores in assessing SpA activity, but with a lower fidelity of the inflammatory markers alone.

Fig. 2 shows the ROC curves of the three composite indices and inflammatory markers as aforementioned.

Optimal cut-off values for ASDAS indicating high disease activity were calculated using ROC 


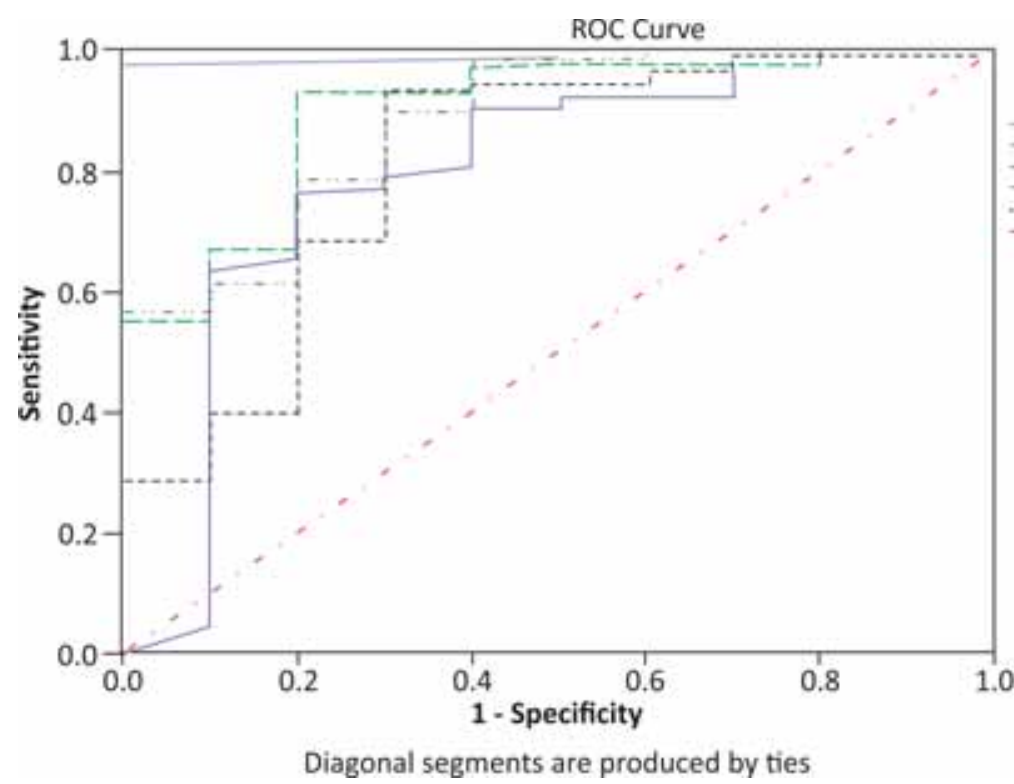

FIGURE 2. ROC curves of disease composite indices and inflammatory markers analysis. Therefore, a value of ASDAS-CRP $>2.6$ had a sensitivity of $89 \%$ and a specificity of $80 \%$. For ASDAS-ESR, a value over 2.5 had a sensitivity of $89 \%$ and a specificity of $70 \%$.

When dividing patients according to BASDAI score ( $>4$ high disease activity; $<4$ low disease activity), $14 \%$ showed a more active disease than the rest of $86 \%$ who had low disease activity. Mean ASDAS scores in the first group were 3.31 and 3.16, respectively, as detailed in Table 6.

TABLE 6. Disease activity scores in patients with high and low disease activity according to BASDAI

\begin{tabular}{|l|c|c|c|}
\hline Variable & $\begin{array}{c}\text { High disease } \\
\text { activity } \\
\text { (BASDAl }>4, \mathrm{n}=14)\end{array}$ & $\begin{array}{c}\text { Low disease } \\
\text { activity } \\
(\text { BASDAl }<5, \mathrm{n}=86)\end{array}$ & $\mathbf{P}$ \\
\hline ASDAS-CRP & $3.31 \pm 1.34$ & $1.41 \pm 0.75$ & $<0.001$ \\
\hline ASDAS-ESR & $3.16 \pm 1.32$ & $1.41 \pm 0.71$ & $<0.001$ \\
\hline $\mathrm{CRP}(\mathrm{mg} / \mathrm{l})$ & $42.39 \pm 45.88$ & $7.08 \pm 9.82$ & $<0.001$ \\
\hline $\mathrm{ESR}(\mathrm{mm} / \mathrm{h})$ & $41.5 \pm 31.12$ & $13.82 \pm 14.84$ & $<0.001$ \\
\hline
\end{tabular}

Both ASDAS scores showed good discriminatory value with a SMD of 2.1587 for ASDAS-CRP $(95 \%$ CI 1.4822-2.8351) and 1.8503 for ASDAS-ESR (95\% CI 1.1922-2.5084) when relating to BASDAI.

When correlating disease activity scores for the entire study group $(\mathrm{n}=100)$ we observed that BASDAI correlated to both ASDAS scores $(r=0.65$ and $0.71, \mathrm{P}<0.001)$ and that PtGA correlated both to disease activity scales, with a stronger correlation to BASDAI $(r=0.912, \mathrm{P}<0.01)$ than ASDAS $(r=0.67$ and $0.71, \mathrm{P}<0.01$ for both) and to acute phase reactants.
Mean values for the variables of interest for the study group are shown in Table 7.

TABLE 7. Mean values of disease activity variables in SpA in the study group $(n=100)$

\begin{tabular}{|l|l|}
\hline Variable & Mean \pm standard deviation SD \\
\hline BASDAI & $1.78 \pm 1.79$ \\
\hline ASDAS-CRP & $1.65 \pm 1.04$ \\
\hline ASDAS-ESR & $1.63 \pm 0.99$ \\
\hline ESR & $17.25 \pm 19.69$ \\
\hline CRP & $11.45 \pm 21.5$ \\
\hline PtGA & $1.93 \pm 1.88$ \\
\hline
\end{tabular}

\section{DISCUSSIONS}

All the existing tools ranging from inflammatory markers to disease activity scores orient the clinician to the intensity of the disease activity. Among the latter, BASDAI is the most widely used tool for measuring SpA activity. However it can only partially reflect disease intensity because it is entirely patient-oriented and it does not encompass individual clinical manifestations. On the other hand ASDAS score combines patient measures with acute phase reactants therefore recent studies proposed using ASDAS in order to differentiate various levels of disease activity $(14,17,18)$.

Our study compared the performance of disease activity scores when applying them to a cohort of SpA patients on anti-TNF therapy. We found good correlations between BASDAI and the two ASDAS variants but also between ASDAS and inflammatory markers such as CRP and ESR. All scores had good 
discriminatory values. When using BASDAI as criterion, ASDAS-CRP had better discriminatory power than ASDAS-ESR or acute phase reactants. Its discriminatory capacity was similar to results of other studies $(14,15)$. However when applying the PtGA as variable, BASDAI outperformed ASDAS scores, showing the importance of patients' own evaluation of the disease. These results are in accordance with other published studies, including that of Van der Heijde et al. that states that the only situation in which BASDAI can surpass ASDAS scores was when assessing whether the patient is or not in an acceptable symptom state (PASS patient acceptable symptom state) $(15,16,19)$.

Until their certified role in radiographic progression, the use of NSAIDs in patients with SpA undergoing biologic therapy is linked to an increased disease activity. This theory was highlighted in the present study that showed a higher intake in patients with active disease.

Disease activity in $\mathrm{SpA}$ patients can indeed be evaluated through the abovementioned tools, showing good sensibility and specificity rates in our study.

However, more objective tools impose in order to make rational and undoubted treatment decisions. Therefore, an option would be supplementary determination of anti-TNF serum drug levels and antidrug antibodies. These results can help explain the

\section{REFERENCES}

1. R.A. Colbert, M.L. DeLay, E.I. Klenk, G. Layh-Schmitt, From HLA-B27 to spondyloarthritis: a journey through the ER, Immunol. Rev., vol. 233, no. 1, pp. 181-202, Jan. 2010.

2. M. Rudwaleit, D. van der Heijde, R. Landewé, J. Listing, N. Akkoc, J. Brandt, et al, The development of Assessment of SpondyloArthritis international Society classification criteria for axial spondyloarthritis (part II): validation and final selection, Ann. Rheum. Dis., vol. 68, no. 6, pp. 777-83, Jun. 2009.

3. M. Rudwaleit, R. Landewé, D. van der Heijde, J. Listing, J. Brandt, et al, The development of Assessment of SpondyloArthritis international Society classification criteria for axial spondyloarthritis (part I): classification of paper patients by expert opinion including uncertainty appraisal, Ann. Rheum. Dis., vol. 68, no. 6, pp. 770-6, Jun. 2009

4. O. Akgul, S. Ozgocmen, Classification criteria for spondyloarthropathies, World J. Orthop., vol. 2, no. 12, pp. 107-15, Dec. 2011

5. J. Sieper, Developments in therapies for spondyloarthritis, Nat Rev. Rheumatol., vol. 8, no. 5, pp. 280-7, May 2012.

6. J. Paccou, E. Solau-Gervais, E. Houvenagel, J. Salleron, H. Luraschi, P. Philippe, B. Duquesnoy, R.-M. Flipo, Efficacy in current practice of switching between anti-tumour necrosis factor- $a$ agents in spondyloarthropathies, Rheumatology (Oxford)., vol. 50, no. 4, pp. 714-20, Apr. 2011.

7. M. Svenson, P. Geborek, T. Saxne, K. Bendtzen, Monitoring patients treated with anti-TNF-alpha biopharmaceuticals: assessing serum infliximab and anti-infliximab antibodies, Rheumatology (Oxford)., vol. 46, no. 12, pp. 1828-34, Dec. 2007. subsequent mechanisms of non-response in patients with active disease and they can reflect the disease status, in addition to conventional scores. A satisfactory drug serum level by standardized cut-offs can lead to dose lowering or administration spacing, without risking a disease flare. Thus, future clinical approach might lead to a personalized patient scheme with individualized dose and interval administration, with probable economic impact.

One of the limitations of our single-centre study was that the performance of disease activity scores was evaluated only on SpA patients under anti-TNF therapy.

\section{CONCLUSIONS}

This study is a "snapshot" of SpA patients undergoing anti-TNF treatment at a certain point in their disease evolution, with overall activity evaluation. In our research we proved that disease activity scores have good discriminatory powers. BASDAI surpassed ASDAS scores when relating to the PtGA but ASDAS-CRP might be leading the path to a more objective evaluation that can differentiate patients with high or low disease activity. However, in order to prove ASDAS feasibility we suggest further prospective longitudinal studies on larger SpA populations with various degrees of disease activity.

8. G.M. Bartelds, Development of Antidrug Antibodies Against Adalimumab and Association With Disease Activity and Treatment Failure During Long-term Follow-up, JAMA, vol. 305, no. 14, p. 1460, Apr. 2011.

9. G.J. Wolbink, M. Vis, W. Lems, A.E. Voskuyl, E. de Groot, M. T. Nurmohamed, S. Stapel, P.P. Tak, L. Aarden, B. Dijkmans, Development of antiinfliximab antibodies and relationship to clinical response in patients with rheumatoid arthritis, Arthritis Rheum., vol. 54, no. 3, pp. 711-5, Mar. 2006.

10. M. Jani, H. Chinoy, R.B. Warren, C. E.M. Griffiths, D. Plant, B. Fu, A.W. Morgan, A.G. Wilson, J.D. Isaacs, K.L. Hyrich, A. Barton, Clinical utility of random anti-TNF drug level testing and measurement of anti-drug antibodies on long-term treatment response in rheumatoid arthritis, Arthritis Rheumatol., vol. 67, no. 8 , p. n/a-n/a, Apr. 2015

11. E.L. Kneepkens, J.C.-C. Wei, M.T. Nurmohamed, K.-J. Yeo, C.Y. Chen, I.E. van der Horst-Bruinsma, D. van der Kleij, T. Rispens, G. Wolbink, C.L.M. Krieckaert, Immunogenicity, adalimumab levels and clinical response in ankylosing spondylitis patients during 24 weeks of follow-up, Ann. Rheum. Dis., vol. 74, no. 2, pp. 396-401, Mar. 2015.

12. E.L. Kneepkens, C.L.M. Krieckaert, D. van der Kleij, M.T. Nurmohamed, I.E. van der Horst-Bruinsma, T. Rispens, G.J. Wolbink, Lower etanercept levels are associated with high disease activity in ankylosing spondylitis patients at 24 weeks of follow-up, Ann. Rheum. Dis., vol. 74, no. 10, pp. 1825-9, Oct. 2015.

13. W. Afif, E.V. Loftus, W.A. Faubion, S.V. Kane, D.H. Bruining, K.A. Hanson, W.J. Sandborn, Clinical utility of measuring 
infliximab and human anti-chimeric antibody concentrations in patients with inflammatory bowel disease, Am. J. Gastroenterol., vol. 105, no. 5, pp. 1133-9, May 2010.

14. A. Spoorenberg, A. van Tubergen, R. Landewé, M. Dougados, S. van der Linden, H. Mielants, H. van de Tempel, D. van der Heijde, Measuring disease activity in ankylosing spondylitis: patient and physician have different perspectives, Rheumatology (Oxford)., vol. 44, no. 6, pp. 789-95, Jun. 2005.

15. D. van der Heijde, E. Lie, T.K. Kvien, J. Sieper, F. Van den Bosch, J. Listing, J. Braun, R. Landewé, ASDAS, a highly discriminatory ASAS-endorsed disease activity score in patients with ankylosing spondylitis, Ann. Rheum. Dis., vol. 68, no. 12, pp. 1811-8, Dec. 2009.

16. M. Godfrin-Valnet, M. Puyraveau, C. Prati, D. Wendling, Flare in spondyloarthritis: Thresholds of disease activity variations., Joint. Bone. Spine, vol. 82, no. 3, pp. 192-5, May 2015.

17. S.Z. Aydin, M. Can, P. Atagunduz, H. Direskeneli, Active disease requiring TNF-alpha-antagonist therapy can be well discriminated with different ASDAS sets: a prospective, follow-up of disease activity assessment in ankylosing spondylitis, Clin. Exp. Rheumatol., vol. 28, no. 5, pp. 752-5, Jan 2015.

18. M. Xu, Z. Lin, X. Deng, L. Li, Y. Wei, Z. Liao, Q. Li, Q. Wei, Z. Hu, Y. Zhang, Q. Lin, J. Huang, T. Li, Y. Pan, Y. Wu, O. Jin, B. $\mathrm{Yu}$, and J. Gu, The Ankylosing Spondylitis Disease Activity Score is a highly discriminatory measure of disease activity and efficacy following tumour necrosis factor- $\alpha$ inhibitor therapies in ankylosing spondylitis and undifferentiated spondyloarthropathies in China, Rheumatology (Oxford)., vol. 50, no. 8, pp. 1466-72, Aug. 2011.

19. E. Kilic, G. Kilic, O. Akgul, S. Ozgocmen, Discriminant validity of the Ankylosing Spondylitis Disease Activity Score (ASDAS) in patients with non-radiographic axial spondyloarthritis and ankylosing spondylitis: a cohort study, Rheumatol. Int., vol. 35, no. 6, pp. 981-9, Jun. 2015. 